\title{
Photostimulated desorption performance of the future circular hadron collider beam screen
}

\author{
L. A. González®, V. Baglin, P. Chiggiato, C. Garion, and R. Kersevan๑ \\ CERN, The European Organization for Nuclear Research, CH-1211 Geneva, Switzerland \\ S. Casalbuoni॰ \\ European XFEL, Holzkoppel 4, 22869 Schenefeld, Germany \\ and Institute for Beam Physics and Technology, Karlsruhe Institute of Technology, \\ Hermann-von-Helmholtz-Platz 1, D-76344 Eggenstein-Leopoldshafen, Germany \\ A. Grau and D. Saez de Jaureguiఠ \\ Institute for Beam Physics and Technology, Karlsruhe Institute of Technology, \\ Hermann-von-Helmholtz-Platz 1, D-76344 Eggenstein-Leopoldshafen, Germany \\ I. Bellafont and F. Pérez \\ ALBA Synchrotron Light Source, Barcelona 08290, Spain
}

(Received 22 February 2021; revised 11 October 2021; accepted 25 October 2021; published 10 November 2021)

\begin{abstract}
Synchrotron radiation (SR) originated at superconducting bending magnets is known to be at the origin of several beam detrimental effects related to vacuum instabilities. One of the major challenges in the design of the vacuum beam pipes of high-energy hadron colliders is the SR coping strategy. In the case of the future circular hadron collider (FCC-hh), a Cu-coated beam screen (BS) operating in the range of $40-60 \mathrm{~K}$ has been designed with the aim of protecting the superconducting magnet cold bores from direct synchrotron irradiation. In order to experimentally study the FCC-hh BS vacuum and cryogenic performance, two sample prototypes were manufactured and installed in the beam screen test-bench experiment (BESTEX) at the Karlsruhe Research Accelerator (KARA) at the Karlsruhe Institute of Technology (KIT). The emitted SR has a critical energy of $6.2 \mathrm{keV}$, very similar to the $4.6 \mathrm{keV}$ of FCC-hh. Irradiation at both room (RT) and cryogenic $(77 \mathrm{~K})$ temperatures showed a significant reduction of the molecular photostimulated desorption yields $(\eta)$ of the FCC-hh beam screen compared to those of $\mathrm{Cu}$ samples. A first approximation of $\eta$ and its evolution with the photon dose accumulated on the FCC-hh BS prototype at $77 \mathrm{~K}$ allows to estimate that a machine conditioning period of $\sim 1.2$ months would be needed to reduce the photostimulated molecular density at the necessary levels to ensure a $100 \mathrm{~h}$ beam lifetime at nominal FCC-hh operation.
\end{abstract}

DOI: $10.1103 /$ PhysRevAccelBeams.24.113201

\section{INTRODUCTION}

The future circular collider (FCC) is a study that aims at developing a conceptual design for the large hadron colliders (LHC's) successor. The FCC-hh is a $100 \mathrm{~km}$ hadron collider foreseen to achieve unprecedented collision energies of $100 \mathrm{TeV}$ at center of mass [1-3]. For such energies, the proton beams circulating along the arcs of the machine generate levels of beam-induced synchrotron

\footnotetext{
*luis.gonzalez.gomez@cern.ch

Published by the American Physical Society under the terms of the Creative Commons Attribution 4.0 International license. Further distribution of this work must maintain attribution to the author(s) and the published article's title, journal citation, and DOI.
}

radiation (SR) orders of magnitude larger than in the LHC $[1,4]$. A comparison of the main SR-related parameters of FCC-hh and LHC is shown in Table I. Similarly to the case of the LHC, in order to reduce the cryogenic power consumption and to guarantee vacuum stability, FCChh's vacuum chamber design necessitates a beam screen (BS) to intercept the SR emitted by the beam before it strikes the magnet cold bore at $1.9 \mathrm{~K}$. Such BS is a complex technological component which has been designed with a number of different functionalities that should all be simultaneously fulfilled to achieve the necessary conditions of vacuum pressure, temperature, and electron cloud (EC) density. The pressure in the vacuum chamber during machine operation must be controlled in order to keep the beam-gas scattering lifetime higher than $100 \mathrm{~h}$ [5]. Specifically, a hydrogen equivalent molecular gas density 
TABLE I. Comparison of the LHC's and FCC-hh's relevant baseline parameters [1].

\begin{tabular}{lcc}
\hline \hline & LHC & FCC-hh \\
\hline Beam energy [TeV] & 7 & 50 \\
Circumference [km] & 26.7 & 100 \\
Dipole magnetic field [T] & 8.3 & 15.9 \\
SR photon flux [ph ${ }^{-1} \mathrm{~s}^{-1}$ ] & $1 \times 10^{17}$ & $1.7 \times 10^{17}$ \\
SR power [W/m] & 0.22 & 35.4 \\
SR critical energy [eV] & 43.8 & 4286.3 \\
\hline \hline
\end{tabular}

(MD) of $1 \times 10^{15} \mathrm{H}_{2 \mathrm{eq}} / \mathrm{m}^{3}$ has been estimated, similarly to the one of LHC, as the maximum value to ensure the machine's operation [6,7]. Photostimulated desorption (PSD), in this respect, is the most important contribution to the MD in the BS of the FCC-hh.

In such framework, the necessity of studying the vacuum and cryogenic performance of the proposed FCC-hh BS arises as a key step for its design validation. To that end, two identical sample prototypes have been manufactured in compliance with the baseline design of the FCC-hh BS $[5,8]$. The samples have been tested at the beam screen test-bench experiment (BESTEX) installed in the $2.5 \mathrm{GeV}$ electron storage ring Karlsruhe Research Accelerator (KARA) light source at KIT. KARA has been chosen due to its similarities with the FCC-hh in terms of SR spectrum, photon flux, and critical energy $\left(\varepsilon_{c}\right)$ [9], defined as the energy at which the SR power spectrum is divided into two equal parts. Due to such similarities, the irradiation of the BS prototypes at BESTEX makes it possible to obtain empiric confirmation of the functionality of the BS designs. Moreover, the simulation techniques used to estimate the vacuum performance during operation of FCC-hh can be benchmarked with the data obtained at BESTEX.

\section{EXPERIMENTAL}

The experiments were carried out at the setup BESTEX installed in the $2.5 \mathrm{GeV}$ electron storage ring KARA light source at KIT.
BESTEX is an experimental instrument that allows to study SR-induced effects on nonleak tight tubular samples under ultrahigh vacuum (UHV) conditions. A schematic layout of BESTEX is presented in Fig. 1. SR originated at KARA's bending magnet can be cropped at the slits, both vertically and horizontally, before impinging on the installed BS prototype. The setup can be pivoted about a vertical axis in order to be able to irradiate at any required glancing angle. Two 2000 l/s hydrogen pumping speed NexTorr NEG cartridge pumps are placed at each extremity of the sample under study providing a symmetric longitudinal pressure profile. Each cartridge counts on a $40 \mathrm{l} / \mathrm{s}$ nominal pumping speed ion pump to allow $\mathrm{CH}_{4}$ and rare gas evacuation.

Calibrated Bayard-Alpert Gauges (BAG) are strategically placed along the system. One of such BAG's and a calibrated residual gas analyzer (RGA) [11,12] allows to measure photo-desorption yields from the inner part of the sample under study by using a chimney connection as depicted in Fig. 1. Further description of BESTEX can be found elsewhere [10]. The sample under study is a $2 \mathrm{~m}$ long prototype manufactured in compliance with the FCC-hh BS baseline design [5,8] (see Fig. 2). It is produced from formed and welded high $\mathrm{Mn}$, high $\mathrm{N}$ grade austenitic stainless steel strip. This grade ensures a low-magnetic permeability at operating temperature while offering good mechanical properties. It implements cooling pipes produced with the same stainless steel grade. The seamless tubes are obtained by cold drawing and subsequent rollforming to get the required "half-moon" shape. The internal wall (main chamber) is laser welded with the cooling tubes. Then, the beam screen is assembled before the laser welding of the external wall onto the cooling tubes. It also implements a main chamber (MC) and an antechamber (AC) separated by two "U"-bent plates facing each other and separated by a long vertical slot at the BS's equatorial plane, coincident to the plane of the proton orbit. A $0.3 \mathrm{~mm}$ thick copper layer must be applied onto the inner walls of $\mathrm{MC}$ and $\mathrm{AC}$ in order to reduce electrical impedance during machine operation. SR then travels through the vertical slot

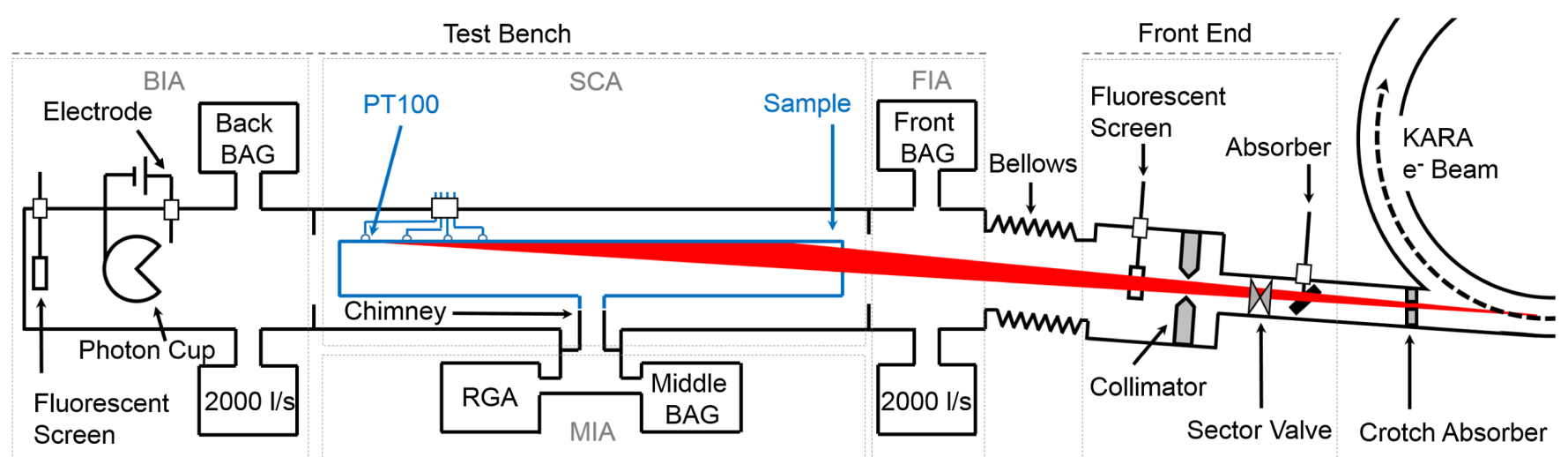

FIG. 1. Schematic layout of BESTEX [10]. 


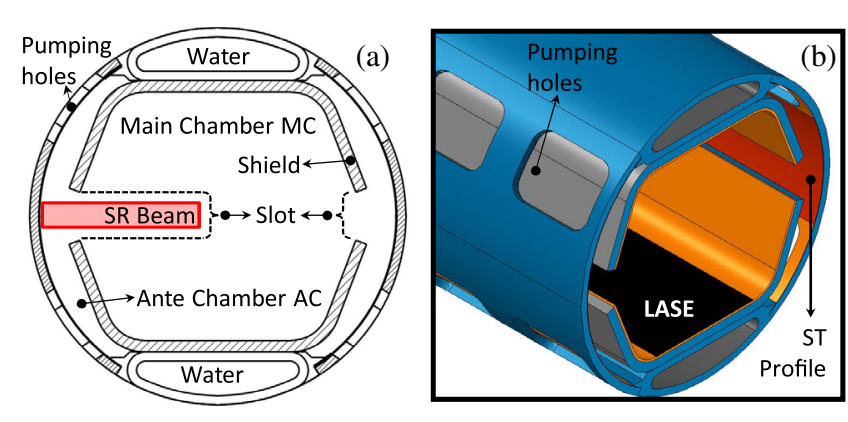

FIG. 2. Graphic descriptions of the BS design in which compliance the sample presented in this work were manufactured. (a) Cross section of BS. The red square represents the area of the SR beam. (b) 3D representation of BS in which the disposition of the sawtooth profile, LASE surface and pumping holes are visible.

before impinging on a sawtooth (ST) profile which consists of an engraved geometry featuring a pattern of consecutive teeth of nominal $40 \mu \mathrm{m}$ of height and $200 \mu \mathrm{m}$ pitch [13]. The orientation of the ST is such that SR impinges onto the short teeth face with an angle close to $90^{\circ} \mathrm{C}$, enhancing in this way the SR absorption at the AC inner surface. The design features a series of pumping holes providing a geometrical transparency of $9.3 \%$. They are located at the $\mathrm{AC}$ allowing the molecules desorbed during machine operation to be efficiently pumped by the surrounding $1.9 \mathrm{~K}$ superconducting magnet cold bore. At the upper and lower walls of $\mathrm{MC}$, a laser ablated surface engineering (LASE) [14] treatment is applied, proposed as anti EC solution alternative to amorphous carbon coating (a-C) [15-18]. Such treatment, which covers $15 \%$ of the entire BS inner surface, modifies the surface at the micro- and nanometric scale providing a drastic reduction of the surface's secondary electron yield down to values lower than 1 in comparison to the 1.1 of a-C [19-21], and hence minimizing the impact of EC effects during machine operation. The scalability and budgetary viability of applying LASE is still under study. Before insertion into BESTEX, each sample was cleaned following standard UHV procedures [22]. The whole setup was then aligned with respect to the KARA's electron beam plane. During the alignment procedure, the setup is placed so as to accurately irradiate the intended sample area with an error of $\pm 200 \mu \mathrm{m}$.

The experimental data here presented correspond to the irradiation of two identical FCC-hh BS prototypes, BS1 and BS2, respectively. BS1 was kept at RT while BS2 was cooled down to $77 \mathrm{~K}$, by using water and LN2 as coolant, respectively. Before BS1 at RT, a standard bakeout cycle of $24 \mathrm{~h}$ at $150^{\circ} \mathrm{C}$ was performed in order to remain within the vacuum pressure limits required to operate the storage ring KARA. In the case of BS2, bakeout cycles were not necessary due to the background pressure reduction inherent to the cold sample's surface.

\section{RESULTS}

The partial pressure rise of $\mathrm{H}_{2}, \mathrm{CH}_{4}, \mathrm{CO}$, and $\mathrm{CO}_{2}$ originated by means of PSD inside the FCC-hh BS prototype was monitored during irradiation of BS1 and BS2, at RT and $77 \mathrm{~K}$, respectively, in BESTEX. The molecular PSD yield $(\eta)$, i.e. average number of molecules desorbed per incident photon depends on the gas species, on the chemical state and on the morphology of the irradiated surface [23]. For each specific vacuum system, $\eta$ can be obtained from the photostimulated pressure rise $\Delta P$ and the system's effective pumping speed $S_{\text {eff }}$ :

$$
\eta=\frac{\Delta P \times S_{\mathrm{eff}}}{\Gamma \times k T}
$$

where $\Gamma$ is the SR photon flux, $k$ is the Boltzman's constant, and $T$ is the system's temperature. The value of $S_{\text {eff }}$, which depends on the system's geometry, is highly affected by the vacuum conductance $C$ to which the desorbed molecules are subjected before being pumped and is given by

$$
1 / S_{\text {eff }}=1 / S+1 / C
$$

where $S$ is the known pumping speed of the vacuum pumps that eventually evacuate the desorbed molecules from the system. The FCC-hh BS is characterized by a high diameter-to-length aspect ratio and the presence of pumping holes longitudinally distributed. The latter makes the determination of $C$ a not straightforward task and hence, the use of Eq. (2) an unsuitable solution to analytically calculate $S_{\text {eff }}$. For this reason, the Montecarlo test particle simulation software Molflow+ [24] was used to model BESTEX together with the $2 \mathrm{~m}$ FCC-hh BS prototype in their experimental configuration to estimate the values of $S_{\text {eff }}$ for each gas, allowing to calculate $\eta$ according to the observed SR-induced partial pressure rise. Table II presents the values of $S_{\text {eff }}$ used in this work for each gas. In the case of $\mathrm{CH}_{4}$ and $\mathrm{CO}_{2}$, a sample's sticking probability of 0.03 and 0.003 was assumed, respectively, for the calculation of $S_{\text {eff }}$ at $77 \mathrm{~K}$. For the other gas species, the sample's sticking probability at $77 \mathrm{~K}$ was assumed to be negligible. Such sticking coefficient values were estimated in agreement with Ref. [25], experimentally

TABLE II. Effective pumping speeds at BESTEX in the presence of the FCC-hh BS prototype calculated with Molflow + for each gas.

\begin{tabular}{lrr}
\hline \hline & \multicolumn{2}{c}{ Temperature $[\mathrm{K}]$} \\
\cline { 2 - 3 } Gas species & 300 & 77 \\
\hline $\mathrm{H}_{2}$ & 500 & 500 \\
$\mathrm{CH}_{4}$ & 100 & 300 \\
$\mathrm{CO}$ & 200 & 200 \\
$\mathrm{CO}_{2}$ & 100 & 1400 \\
\hline \hline
\end{tabular}




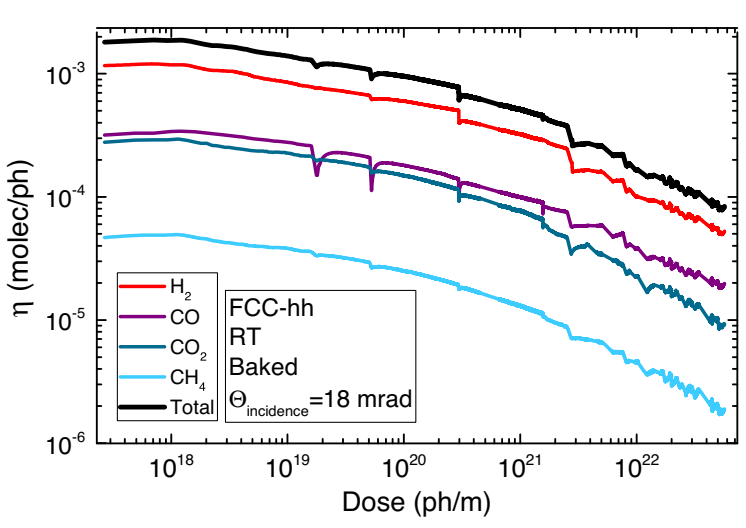

FIG. 3. Evolution of $\eta$ presented for $\mathrm{H}_{2}$ (red line), $\mathrm{CO}$ (purple line), $\mathrm{CO}_{2}$ (green line), and $\mathrm{CH}_{2}$ (blue line) as a function of the $18 \mathrm{mrad}$ photon dose accumulated on the FCC-hh BS prototype at RT. Black line represents the evolution of the total molecular yield obtained by summing up the contribution of each gas specie.

obtained standard oxygen-free electronic (OFE) $\mathrm{Cu}$. It is important to take into account that such $S_{\text {eff }}$ values might be subjected to certain variations due to the presence of LASE which morphology and chemical state is drastically different from that of OFE $\mathrm{Cu}$. Since the sticking coefficient of LASE is unknown to the date of this publication, as a first approximation, any possible modifications of the sticking coefficient due to the presence of LASE have not been taken into account.

Figure 3 shows the values of $\eta$ for the four relevant gas species as a function of the photon beam dose accumulated on the FCC-hh BS at RT.

A decaying evolution of $\eta$ with the accumulated SR dose can be observed as a result of surface conditioning. Such effect is ascribed to the photon-induced gas content depletion at the irradiated areas together with the final evacuation of the desorbed molecules at the vacuum pumps. The pressure rise inside the BS is dominated by the contribution of $\mathrm{H}_{2}$ with a concentration of $\sim 63 \%$ during the whole irradiation run, in agreement with previous experimental findings [23,26-28]. The concentrations of $\mathrm{CO}, \mathrm{CO}_{2}$, and $\mathrm{CH}_{4}$ remained at the rather constant values of $19 \%, 16 \%$, and 3\%, respectively. The black line in Fig. 4 shows the evolution of the total $\eta$ obtained by summing up the contribution of the four gas species. Figure 4 shows a comparison of $\eta_{\mathrm{FCC}-\mathrm{hh}}$ measured at BESTEX $\left(\varepsilon_{c}=6.4 \mathrm{keV}\right)$ with previously published results $[26,29,30]$ on flat (without ST structuring and pumping holes) baked $\mathrm{Cu}$ tubular samples under irradiation at RT and different $\varepsilon_{c}$, namely $3.75 \mathrm{keV}\left(\eta_{3.75 \mathrm{keV}}\right), 2.66 \mathrm{keV}\left(\eta_{2.66 \mathrm{keV}}\right)$, and $0.5 \mathrm{keV}$ $\left(\eta_{0.5 \mathrm{keV}}\right)$. The data have been converted to hydrogen equivalent units $\left(\eta_{\mathrm{H}_{2} \mathrm{eq}}\right)$, allowing to directly assess the performance of each sample with regard to their impact on the vacuum level. The value of $\eta$ for each gas has been transformed according to their equivalence to $\mathrm{H}_{2}$ in terms of the nuclear beam-gas scattering cross section that each

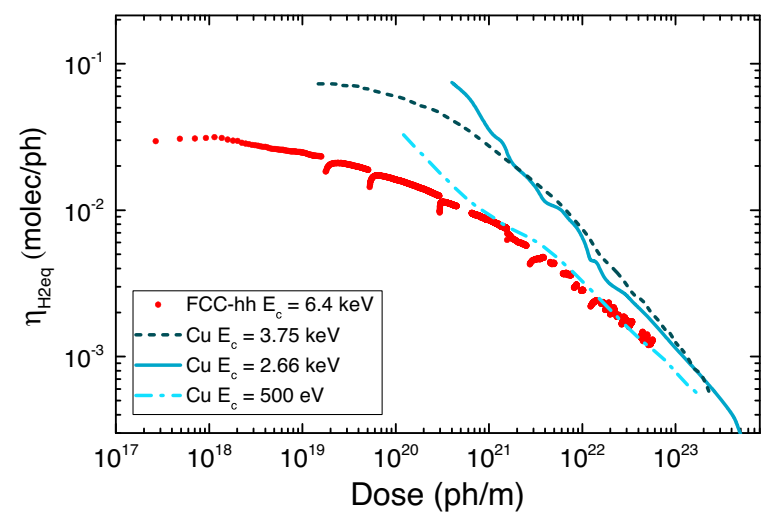

FIG. 4. $\mathrm{H}_{2}$ equivalent molecular PSD yields $\left(\eta_{H_{2 \mathrm{eq}}}\right)$ for baked $\mathrm{Cu}$ at RT as obtained from the bibliography at $\varepsilon_{c}=3.75 \mathrm{keV}$ (dashed line) [23], $\varepsilon_{c}=2.66 \mathrm{keV}$ (solid line) [26], and $\varepsilon_{c}=$ $500 \mathrm{eV}$ (dot-dashed line) [30] compared with the measured yield for FCC-hh (red dots).

different species represents. In order to do so, the following expression, derived elsewhere [7], has been used:

$$
\eta_{\mathrm{H}_{2} \mathrm{eq}}=\eta_{H 2} \sum_{j}\left(\frac{\eta_{j}}{\eta_{H 2}} \frac{\sigma_{j}}{\sigma_{H 2}} \sqrt{\frac{M_{j}}{M_{H 2}}}\right)
$$

where $\sigma_{j}$ and $M_{j}$ represent the nuclear scattering cross section and the molar mass of each gas, respectively. Table III shows the equivalences to $\mathrm{H}_{2}$ for the different gas species used to perform the conversion into $\mathrm{H}_{2}$ equivalent by means of Eq. (3). Figure 4 shows values of $\eta$ of FCC-hh $\left(\eta_{6.4 \mathrm{keV}}\right)$ lower than $\eta_{2.66 \mathrm{keV}}$ and $\eta_{3.75 \mathrm{keV}}$ at all doses. Only for doses higher than $10^{22} \mathrm{ph} / \mathrm{m}, \eta_{\text {FCC-hh }}$ reaches values similar to those measured for $0.5 \mathrm{keV}$. At high doses, it can be noticed that all the curves tend to converge to a similar value for doses of $\sim 1 \times 10^{23} \mathrm{ph} / \mathrm{m}$. The lower values of $\eta$ observed for the FCC-hh BS are ascribed to the effect of the ST profile onto which the first SR impingement takes place. Due to the geometry of the ST profile, the SR impinges normally onto the shortest of each teeth's face. Hence, despite the SR grazing angle in the coarse scale, the PSD processes are subjected to an efficient angle of incidence drastically increased in comparison to that for flat $\mathrm{Cu}$ samples, leading to a reduction of the PSD originated by reflected photons. On the other hand, SR penetrates deeper into the $\mathrm{Cu}$ bulk as the glancing angle increases. Such effect makes the generation of photo- and

TABLE III. Equivalences to $\mathrm{H}_{2}$ of the different gas species.

\begin{tabular}{lcc}
\hline \hline Gas species $(j)$ & $\sigma_{j} / \sigma_{H_{2}}$ & Total $\mathrm{H}_{2_{\mathrm{eq}}}$ \\
\hline $\mathrm{H}_{2}$ & 1 & 1 \\
$\mathrm{CH}_{4}$ & 6.0 & 16.8 \\
$\mathrm{CO}$ & 9.0 & 33.5 \\
$\mathrm{CO}_{2}$ & 13.9 & 64.9 \\
\hline \hline
\end{tabular}




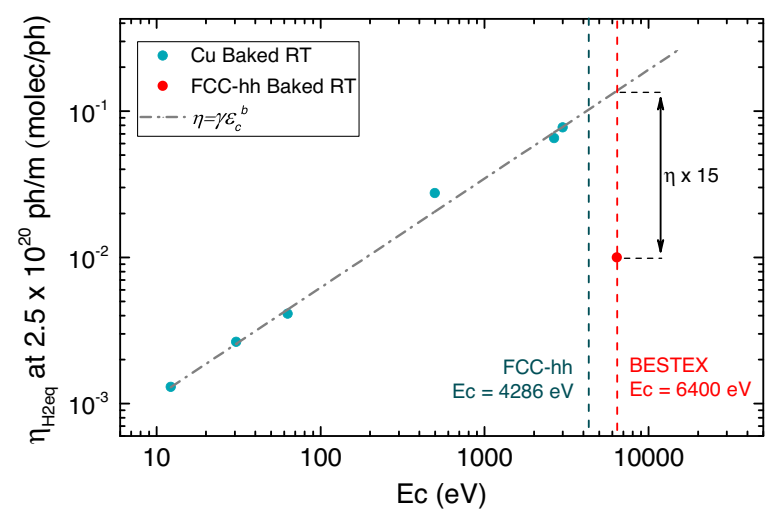

FIG. 5. Blue dots: $\eta_{\text {Heq }}$ dependence on $\varepsilon_{c}$ for RT flat $\mathrm{Cu}$ at a common accumulated photon dose of $2.5 \times 10^{20} \mathrm{ph} / \mathrm{m}$ as calculated from Refs. [23,27]. Dot-dashed line: fit of $\eta_{\mathrm{Heq}}$ as a function of $\varepsilon_{c}$ for RT flat $\mathrm{Cu}$ according to Eq. (2). Red dot: $\eta_{\mathrm{Heq}}$ for FCChh BS at an accumulated photon dose of $2.5 \times 10^{20} \mathrm{ph} / \mathrm{m}$.

secondary electrons, responsible for PSD phenomena, to take place deeper in the material reducing the amount of molecules desorbed from it. Moreover, the presence of the ST leads to a reduction of the final irradiated surface, since only a fraction of the teeth's shortest face (40 $\mu$ m length) is exposed to the SR for each $200 \mu \mathrm{m}$ teeth pitch along the $\mathrm{BS}$, reducing the efficient area where PSD processes take place [5].

It is well known that $\eta$ scales linearly with $\varepsilon_{c}$ according to the following empirical expression $[23,27]$ :

$$
\eta=\alpha \varepsilon_{c}^{b}
$$

where $\alpha$ and $b$ are positive constants that depend on the desorbed gas and the irradiated material. Blue dots in Fig. 5 show the results, in hydrogen equivalent units, presented in Refs. [26,27] corresponding to measurements on flat baked $\mathrm{Cu}$, irradiated at different $\varepsilon_{c}$, ranging from $10 \mathrm{eV}$ to $3 \mathrm{keV}$ at a glancing angle of $11 \mathrm{mrad}$. The data were extracted in each case at a common dose of $2.5 \times 10^{20} \mathrm{ph} / \mathrm{m}$ which corresponds to $\sim 1 \mathrm{~h}$ of FCC-hh nominal operation. The data have been fitted according to Eq. (4) as represented by the grey dashed line in Fig. 5. The value of $\eta_{\mathrm{FCC}-\text { hh }}$ at KARA's $\varepsilon_{c}$ is also shown. It can be clearly observed that the measured value of $\eta_{\text {FCC-hh }}$ is about 15 times lower than the value for flat $\mathrm{Cu}$ expected from the shown dependence of the data from literature. An even lower value is expected for the real machine, with a slightly lower $\varepsilon_{c}$. Such finding clearly shows that the baseline of the FCC-hh BS design efficiently reduces the PSD contribution to the total MD increase inside the machine's vacuum chamber with respect to its flat counterpart.

A setup upgrade carried out before the sample irradiation at $77 \mathrm{~K}$ allowed to reduce the SR glancing angle down to $3 \mathrm{mrad}$. Consequentially, the resemblance of the irradiating conditions of FCC-hh BS at BESTEX to those expected for
TABLE IV. Comparison of the SR parameters of BESTEX (for the two main configuration of this specific work) and the FCC-hh relevant baseline parameters.

\begin{tabular}{lccc}
\hline \hline & BESTEX & BESTEX & FCC-hh \\
\hline Temperature [K] & 300 & 77 & $40-60$ \\
Glancing angle [mrad] & 18 & 3 & 1.35 \\
Critical energy [keV] & 6.2 & 6.2 & 4.3 \\
SR flux [ph/s/m] & $4.84 \times 10^{16 \mathrm{a}}$ & $1.59 \times 10^{16 \mathrm{a}}$ & $1.7 \times 10^{17}$ \\
SR power [W/m] & 32 & 5.7 & $32^{\mathrm{b}}$ \\
\hline \hline
\end{tabular}

${ }^{\mathrm{a} C}$ Calculated at $130 \mathrm{~mA}$ and $2.5 \mathrm{GeV}$ KARA's beam current and energy, respectively.

${ }^{\mathrm{b}}$ Power received at the BS.

${ }^{\mathrm{c}}$ Average value. Power ranges between $21 \mathrm{~W} / \mathrm{m}$ and $42 \mathrm{~W} / \mathrm{m}$.

the real machine is maximized: Table IV shows the SR parameters of BESTEX during irradiation at RT and $77 \mathrm{~K}$ in comparison with those expected for FCC-hh. It can be seen that both SR flux and power are reduced when going from 18 to $3 \mathrm{mrad}$. This is due to the fact that a specific SR beam geometry was required at each setup orientation. Hence, in order to irradiate within the limits of the samples inner walls, the SR collimator aperture must be reduced for smaller glancing angles. Such discrepancies are not considered to be significant for PSD studies for the following reasons. On one hand, PSD is known to depend on the impinging SR energy and not on its power density [23]. On the other hand, the flux of SR photons arriving to the sample will affect only the conditioning time and not the dependence of $\eta$ with the accumulated photon dose. Hence, despite the discrepancies observed in terms of SR flux and power presented in Table IV, the irradiation conditions at $77 \mathrm{~K}$ have been considered to be representative for the real machine vacuum system. The values for the photon flux at both configurations of BESTEX presented in Table IV were calculated by using the software Synrad [24]. The calculations were performed taking into account KARA's bending magnet characteristics and a stored electron beam of $130 \mathrm{~mA}$ and $2.5 \mathrm{GeV}$. For each geometrical configuration of BESTEX, the corresponding SR flux through the slits was obtained. The final value of the SR flux is subjected to the slits positioning error in the slits [10] lead to an uncertainty in the accumulated photon dose of $1 \%$. A spectral density plot of the white light spectrum generated at KARA's bending magnet can be found in Ref. [10].

The lower panel of Fig. 6 shows the evolution of $\eta$ for each gas as a function of the photon dose accumulated on BS2 at $77 \mathrm{~K}$ at $3 \mathrm{mrad}$. During the acquisition of the data presented in Fig. 6, the sample was also momentarily irradiated at glancing angles different from $3 \mathrm{mrad}$. Since the dependence of $\eta$ with the glancing angle is out of the scope of this study, only data obtained under irradiation at $3 \mathrm{mrad}$ are shown. The latter explains the regions with no data visible at doses $>5 \times 10^{21} \mathrm{ph} / \mathrm{m}$. In the $77 \mathrm{~K}$ case, the pressure rise generated by photon impingement is 


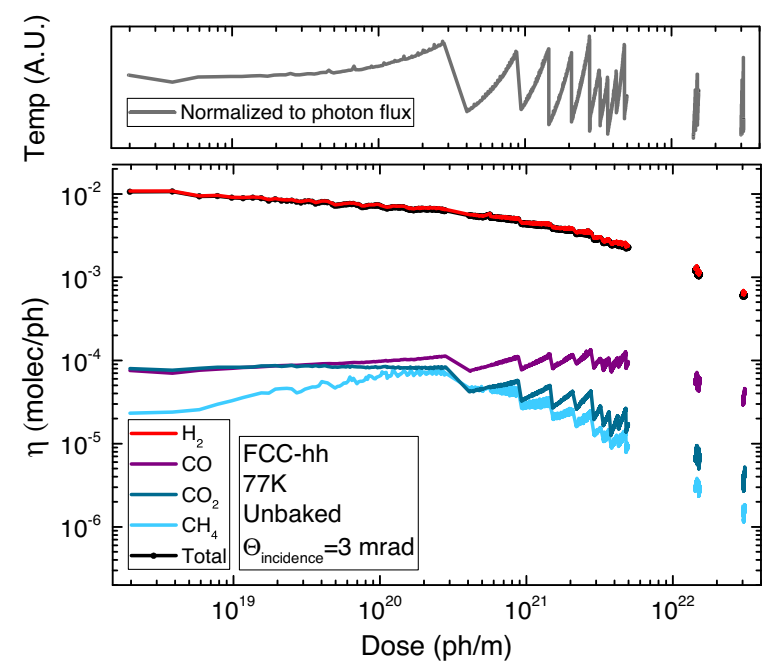

FIG. 6. Upper panel: evolution with the accumulated photon dose of the sample's temperature normalized to the absorbed photon flux. Lower panel: Evolution of $\eta$ presented for $\mathrm{H}_{2}$ (red line), $\mathrm{CO}$ (purple line), $\mathrm{CO}_{2}$ (green line) and $\mathrm{CH}_{4}$ (blue line) as a function of the accumulated 3 mrad photon dose accumulated on the FCC-hh BS prototype at $77 \mathrm{~K}$. Black line represents the evolution of the total molecular yield obtained by summing up the contribution of each gas.

dominated by $\mathrm{H}_{2}$ molecules with a concentration of $~ 90 \%$ for almost the whole irradiation run. As a consequence, the molecular yield for $\mathrm{H}_{2}$ closely resembles $\eta_{\text {total }}$ along the whole dose range as depicted in Fig. 6. The contributions of $\mathrm{CO}, \mathrm{CO}_{2}$, and $\mathrm{CH}_{4}$ have been drastically reduced in comparison with the RT counterpart. Only at doses higher than $10^{22} \mathrm{ph} / \mathrm{m}$, the concentration of CO slightly increases leading to 85.5 and $13.8 \%$ for $\mathrm{H}_{2}$ and $\mathrm{CO}$, respectively. It is worth noting that the values of $\eta$ presented for BS2 are about one order of magnitude higher than those for BS1. The latter has been ascribed to two phenomena. On one hand, as mentioned in Sec. II, BS2 was not subjected to bake out after insertion into BESTEX. The latter results in an increase in the values of $\eta$ with respect to the unbaked BS1. On the other hand, The demonstrated highly efficient SR absorption capabilities of the ST profile have been assumed to be reduced with the decrease of the overall angle of SR incidence during irradiation of BS2 in comparison to BS1. As discussed in [31] such effect shall be enhanced for very small angles in which case, the interaction of the SR with the ST is limited to the tip of each teeth. The SR absorption capabilities of this specific region of the profile are significantly worsen, with respect to the flat side of the teeth, due to unavoidable shape imperfections derived form the manufacturing technique limitations. Such effect consequently increases the amount of reflected photons into the BS, resulting in photostimulated phenomena from its inner walls.

Similarly as in the RT case, the values of $\eta$ obtained at $77 \mathrm{~K}$ follow an overall decaying trend with the increasing accumulated dose. However, the evolution of $\eta$ for $\mathrm{CO}$, $\mathrm{CO}_{2}$, and $\mathrm{CH}_{4}$ is characterized by cycles showing a gradual increase followed by drastic fall in a saw-like oscillating behavior. Such cycles correspond to the $24 \mathrm{~h}$ periodic operation of KARA whose beam current, working in decaying mode, ranges nominally from $160 \mathrm{~mA}$ after beam injection to $90 \mathrm{~mA}$ before beam dump. Under these circumstances, the BS temperature suffers unavoidable oscillations between 86 and $77 \mathrm{~K}$. The upper panel of Fig. 6 shows the FCC-hh BS temperature during irradiation normalized to $\Gamma$ which is directly proportional to KARA's $e^{-}$beam current. In this case, a similar saw-like oscillating behavior reveals a slower decaying rate for the sample's temperature than for KARA's $e^{-}$beam current. The latter suggests that the observed saw-like oscillations in $\eta$ are induced by thermal desorption, whose effect is not contemplated in Eq. (1). It is noticeable that such thermal effect has an influence on $\mathrm{CH}_{4}$ whose sticking coefficient is expected to be negligible at temperatures above $77 \mathrm{~K}$. In this regard, recent studies [32] showed thermal programmed desorption (TPD) of the gases here studied from LASE surfaces, which are present free of direct irradiation at the top and bottom BS's inner walls. In such case, TPD occurs in a much broader and higher temperature range with respect to what is observed from the flat substrates. Such phenomenon is expected to contribute to the observed saw-like oscillating effect here observed for the values of $\eta$ in the FCC-hh BS. Such observation suggests that the sticking coefficient of the FCC-hh BS inner walls is affected by the presence of LASE and the values presented in Table II have been used here to provide an optimistic approximation of $\eta$. It is important to note that the potential gas pumping and releasing capabilities of LASE may have strong consequences in terms of PSD. For that reason, in order to determine the error of the approximation carried out in this work, the value of the sticking coefficient of LASE surfaces must be obtained by experimental methods.

Figure 7 shows the evolution of $\eta_{\mathrm{H}_{2} \mathrm{eq}}$ as a function of the photon dose accumulated on the FCC-hh BS at $77 \mathrm{~K}$ and $3 \mathrm{mrad}$ in a different scale with respect to Fig. 6 . For doses higher than $1 \times 10^{21} \mathrm{ph} / \mathrm{m}$, the values of $\eta_{\mathrm{H}_{2} \text { eq }}$ show a characteristic [23] decaying power law dependence on the accumulated SR dose. Such data were fitted with a coefficient of determination $R^{2}=0.95$ to find decaying evolution with the accumulated photon dose $D$ as

$$
\eta_{H_{2} e q}=1 \times 10^{9} \times D^{-0.531} .
$$

The values of $\mathrm{H}_{2 \mathrm{eq}}$ MD corresponding to each $\eta_{\mathrm{H}_{2} \mathrm{eq}}$ at nominal FCC-hh operation flux were calculated from the value of $\Delta P$, extracted from Eq. (1) by assuming a value of $S_{\text {eff }}=1125 \mathrm{l} /(\mathrm{s} \mathrm{m})$, in agreement with the calculations performed in Refs. [7,33]. Such value of $S_{\text {eff }}$ for the FCChh BS at $40 \mathrm{~K}$ was obtained by taking into account the pumping effect of the $1.9 \mathrm{~K}$ magnet cold bore through the 


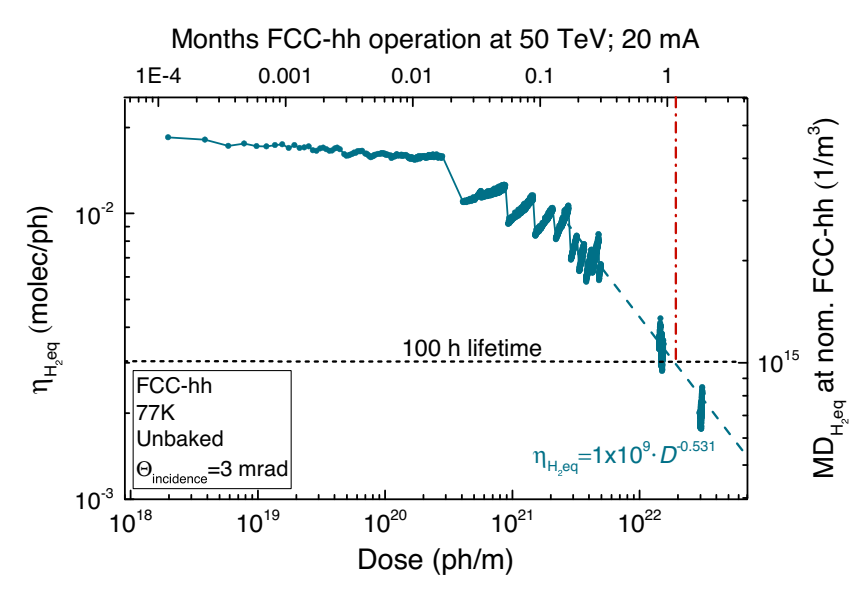

FIG. 7. Evolution of $\eta_{\mathrm{H}_{2} \text { eq }}$ as a function of the $3 \mathrm{mrad}$ glancing photon dose accumulated on the FCC-hh BS prototype at $77 \mathrm{~K}$. Black horizontal dotted line represents the threshold below which the FCC-hh beam lifetime is $>100 \mathrm{~h}$ corresponding to a $\mathrm{H}_{2} \mathrm{eq}$ MD of $1 \times 10^{15} \mathrm{~m}^{-3}$. Dashed line represents the extrapolation of the data according to the observed decaying trend with the accumulated photon dose.

BS's pumping holes. The right axis of Fig. 7 shows the $\mathrm{H}_{2 \mathrm{eq}}$ MD corresponding to each $\eta_{\mathrm{H}_{2} \text { eq }}$ at nominal FCC-hh operation flux. The dashed line in Fig. 7 represents the extrapolation of the experimental data according to Eq. (5). Under these circumstances, the goal of $100 \mathrm{~h}$ nuclear scattering lifetime, corresponding to the previously mentioned gas density of $1 \times 10^{15} \mathrm{H}_{2} / \mathrm{m}^{3}$, would be achieved after a dose of at least $\sim 1.1 \times 10^{22} \mathrm{ph} / \mathrm{m}$ is accumulated. The necessary machine's conditioning time was estimated and represented in the upper axis of Fig. 7. The correlation with the accumulated photon dose was calculated assuming continuous operation at an average beam current of $20 \mathrm{~mA}$, as derived from the case of LHC conditioning period with an average of $\sim 250$ Ah per year over the first 5 years of commissioning up to nominal luminosity [34]. The energy was chosen to be the nominal of FCC-hh as the normal machine's operation routine is to reach full energy and then rising slowly the beam current. As it can be seen from the vertical dashed line in Fig. 7, such conditioning time is equivalent to $\sim 1.2$ months of continuous operation at $20 \mathrm{~mA}$ beam current. Recently published calculations [7] show good agreement with the data here presented in terms of PSD related conditioning time. It is important to notice that only the PSD contribution has been taken into account in this work. However, even though the gas load is expected to be dominated by SR-induced PSD at the FCC-hh BS, the real scenario is as well subjected to electron- and ionstimulated desorption which contribution will, in some extent, increase the conditioning times here estimated.

\section{CONCLUSIONS}

Two FCC-hh BS prototypes have been manufactured in compliance with the current baseline design [5,8], including a ST profile and a LASE surface. The sample was installed on the ad hoc built experimental beamline named BESTEX at the KARA and irradiated at RT and $77 \mathrm{~K}$ under significant levels of SR in order to study its vacuum performance. A total dose of $5 \times 10^{22} \mathrm{ph} / \mathrm{m}$ has been accumulated on the sample at RT during irradiation at $18 \mathrm{mrad}$ glancing angle. The PSD results obtained at RT show that the photostimulated molecular desorption yields of $\mathrm{H}_{2}, \mathrm{CH}_{4}, \mathrm{CO}$, and $\mathrm{CO}_{2}$ have been reduced with the accumulated dose due to surface conditioning effects. The data acquired at BESTEX for the FCC-hh BS prototype at RT were compared with those available in literature for flat $\mathrm{Cu}$ at different critical energies. Such comparison showed that for doses equivalent to $\sim 1 \mathrm{~h}$ of FCC-hh operation, the sample's geometry provides a reduction of the hydrogen equivalent molecular yields by a factor 15 with respect to its flat counterpart. A total dose of $3 \times 10^{22} \mathrm{ph} / \mathrm{m}$ has been accumulated on the sample at $77 \mathrm{~K}$ during irradiation at $3 \mathrm{mrad}$ gracing angle. These conditions have been considered to be close enough to those expected for FCC-hh to assume that the obtained experimental results are representative for the real machine vacuum system. PSD results show that the PSD yield decays following a characteristic power law dependence with the accumulated SR dose. It has been observed that subtle temperature oscillations of the BS inner walls have a notable effect on the MD of the FCC-hh vacuum system suggesting an increase of the BS sticking coefficient originated by the presence of LASE surfaces. Such effect indicates that the sticking coefficient of the FCC-hh BS inner walls is affected by the presence of LASE and the values obtained correspond to an optimistic approximation, hence for completeness, it is of critical importance to experimentally determine the values of the sticking coefficient of LASE in the future. Data extrapolation indicates that, in good agreement with previously published simulation results, a conditioning period of at least 1.2 month is necessary to accumulate the necessary SR dose in order to ensure a PSD-induced MD low enough to provide $100 \mathrm{~h}$ beam lifetime at FCC-hh nominal operation.

\section{ACKNOWLEDGMENTS}

This work was supported by the European Circular Energy-Frontier Collider Study (EuroCirCol) project. EuroCirCol has received funding from the European Union's Horizon 2020 research and innovation programme under Grant No. 654305. The information herein only reflects the views of its authors and the European Commission is not responsible for any use that may be made of the information. The authors wish to thank M. GilCosta for his invaluable coordinating and hands-on contribution to the design, commissioning, assembly, and installation of BESTEX. Also N. Glamann, A. Völker, M. Bank, T. Fischböck, and the KARA technician team from KIT as well as B. Henrist from CERN for the technical 
support; S. Meunier and B. Jenninger from CERN for their support with BAG and RGA calibration; C. Duclos and H. Rambeau for FCC-hh BS protoype manufacturing; A. Gutierrez and J.F. Cortes for the developement of BESTEX's control system; A. Benoit for the contribution in the developement of BESTEX's data acquisition software.

[1] M. Benedikt, European circular energy Frontier Collider Study, H2020-INFRADEV-1-2014-1, 2014.

[2] M. Benedikt et al., FCC-hh: The Hadron Collider: Future Circular Collider Conceptual Design Report Volume 3. Future Circular Collider, Technical Report No. CERNACC-2018-0058, CERN, Geneva, 2018.

[3] M. Benedikt, Q.-Y. Zhang, Q. Wang, Z.-W. Long, and S.-H. Dong, FCC physics opportunities, Eur. Phys. J. C 79, 1 (2019).

[4] M. Benedikt et al., FCC-hh: The hadron collider, Eur. Phys. J. Spec. Top. 228, 755 (2019).

[5] I. Bellafont, M. Morrone, L. Mether, J. Fernández, R. Kersevan, C. Garion, V. Baglin, P. Chiggiato, and F. Pérez, Design of the future circular hadron collider beam vacuum chamber, Phys. Rev. Accel. Beams 23, 033201 (2020).

[6] O. Gröbner, Overview of the LHC vacuum system, Vacuum 60, 25 (2001).

[7] I. Bellafont, L. Mether, R. Kersevan, O. B. Malyshev, V. Baglin, P. Chiggiato, and F. Pérez, Beam induced vacuum effects in the future circular hadron collider beam vacuum chamber, Phys. Rev. Accel. Beams 23, 043201 (2020).

[8] C. Garion, Cold plasma spray application in the FCC, in Proceedings of FCC week17, 2017.

[9] L. A. Gonzalez, Results on the FCC-hh beam screen at the KIT electron storage ring KARA, in Proceedings of IPAC18 (2018).

[10] L. A. Gonzalez, M. Gil-Costa, V. Baglin, P. Chiggiato, C. Garion, R. Kersevan, S. Casalbuoni, E. Huttel, I. Bellafont, and F. Perez, Commissioning of a beam screen test bench experiment with a future circular hadron collider type synchrotron radiation beam, Phys. Rev. Accel. Beams 22, 083201 (2019).

[11] K. Jousten, Pressure measurement with ionization gauges, in CAS_CERN Accelerator School: Vacuum for particle accelerators, 1999.

[12] O. B. Malyshev and K. J. Middleman, In situ ultrahigh vacuum residual gas analyzer "calibration", J. Vac. Sci. Technol. A 26, 1474 (2008).

[13] N. Mahne, V. Baglin, I. R. Collins, A. Giglia, L. Pasquali, M. Pedio, S. Nannarone, and R. Cimino, Photon reflectivity distributions from the lhc beam screen and their implications on the arc beam vacuum system, Appl. Surf. Sci. 235, 221 (2004).

[14] R. Valizadeh, O. B. Malyshev, S. Wang, S. A. Zolotovskaya, W. A. Gillespie, and A. Abdolvand, Low secondary electron yield engineered surface for electron cloud mitigation, Appl. Phys. Lett. 105, 231605 (2014).
[15] C. Y. Vallgren, G. Arduini, J. Bauche, S. Calatroni, P. Chiggiato, K. Cornelis, P. Costa Pinto, B. Henrist, E. Métral, H. Neupert, G. Rumolo, E. Shaposhnikova, and M. Taborelli, Amorphous carbon coatings for the mitigation of electron cloud in the cern super proton synchrotron, Phys. Rev. ST Accel. Beams 14, 071001 (2011).

[16] R. Larciprete, D. Remo Grosso, A. Di Trolio, and R. Cimino, Evolution of the secondary electron emission during the graphitization of thin c films, Appl. Surf. Sci. 328, 356 (2015).

[17] L. A. Gonzalez, R. Larciprete, and R. Cimino, The effect of structural disorder on the secondary electron emission of graphite, AIP Adv. 6, 095117 (2016).

[18] R. Cimino, M. Commisso, D. R. Grosso, T. Demma, V. Baglin, R. Flammini, and R. Larciprete, Nature of the Decrease of the Secondary-Electron Yield by Electron Bombardment and Its Energy Dependence, Phys. Rev. Lett. 109, 064801 (2012).

[19] V. Nistor, L. A. Gonzalez, L. Aguilera, I. Montero, L. Galán, U. Wochner, and D. Raboso, Multipactor suppression by micro-structured gold/silver coatings for space applications, Appl. Surf. Sci. 315, 445 (2014).

[20] M. A. Furman and M. T. F. Pivi, Probabilistic model for the simulation of secondary electron emission, Phys. Rev. ST Accel. Beams 5, 124404 (2002).

[21] G. Rumolo and F. Zimmermann, Practical user guide for ecloud, Technical Report No. CERN-SL-Note-2002-016AP, 2002.

[22] M. Taborelli, Cleaning and surface properties, in CASCERN Accelerator School: Vacuum for particle accelerators, 2017.

[23] O. Gröbner, Dynamic outgassing, Technical Report No. CERN-OPEN-2000-275, 1999.

[24] M. Ady, Monte carlo simulations of ultra high vacuum and synchrotron radiation for particle accelerators, Ph.D. thesis, https://cds.cern.ch/record/2157666.

[25] V. Baglin, I. R. Collins, O. Gröbner, C. Grünhagel, and B. Jenninger, Molecular desorption by synchrotron radiation and sticking coefficient at cryogenic temperatures for h2, ch4, co and co2, Vacuum 67, 421 (2002).

[26] V. Anashin, A. Bulygin, O. Malyshev, L. Mironenko, E. Pyata, D. Krämer, and V. Volkov, Photodesorption and power testing of the sr crotch-absorber for bessy-ii, 1998, https://cds.cern.ch/record/859323.

[27] J. Gómez-Goñi, O. Gröbner, and A. G. Mathewson, Comparison of photodesorption yields using synchrotron radiation of low critical energies for stainless steel, copper, and electrodeposited copper surfaces, J. Vac. Sci. Technol. A 12, 1714 (1994).

[28] O. Grobner, A. G. Mathewson, and P. C. Marin, Extended study of photon stimulated gas desorption from of hc copper by $3.75 \mathrm{kev}$ critical energy photons, In EPAC 92: Third European Particle Accelerator Conference: Berlin, 1992, p. 132.

[29] O. Gröbner, A. G. Mathewson, and P. C. Marin, Gas desorption from an oxygen free high conductivity copper vacuum chamber by synchrotron radiation photons, J. Vac. Sci. Technol. A 12, 846 (1994). 
[30] C. L. Foerster, H. Halama, G. Korn, M. Calderon, and W. Barletta, Desorption measurements of copper and copper alloys for pep-ii, Vacuum 44, 489 (1993).

[31] I. Bellafont, Study of the beam induced vacuum effects in the cryogenic beam vacuum chamber of the Future Circular Hadron Collider, Universitat Politecnica de Catalunya, 2021.
[32] L. Spallino, M. Angelucci, and R. Cimino, Thermal desorption of cryopumped gases from laser treated copper, Phys. Rev. Accel. Beams 23, 063201 (2020).

[33] I. Bellafont, Study of the beam induced vacuum effects in the FCC-hh, Ph.D. thesis, CERN, 2021 (to be published).

[34] V. Baglin, The LHC vacuum system: Commissioning up to nominal luminosity, Vacuum 138, 112 (2017). 